\title{
Acute lymphoblastic leukemia in an adolescent presenting with acute hepatic failure: A case report
}

\author{
RUO-LAN GU, MING XIANG, JING SUO and JING YUAN
}

Department of Hematology, The Third Hospital of Hebei Medical University, Shijiazhuang, Hebei 050051, P.R. China

Received June 26, 2018; Accepted April 22, 2019

DOI: $10.3892 / \operatorname{mco} .2019 .1877$

\begin{abstract}
This case report describes a case of an unusual initial presentation of acute lymphoblastic leukemia in a previously healthy 15 -year-old boy. He initially presented with a 6-day history of tiredness, decreased oral intake, nausea, vomiting, and jaundice (yellow sclera and dark urine) with evidence of acute hepatic failure, presenting as an increase in alanine aminotransferase (ALT)/aspartate aminotransferase (AST)/total bilirubin and a decrease in prothrombin activity. A complete serological evaluation for liver disease was negative. The levels of serum AST and ALT declined following hepatoprotective treatment. Bone marrow biopsy was diagnostic, revealing $68.15 \%$ blasts with markers consistent with acute B-cell lymphoblastic leukemia. This case report emphasizes that acute hepatic failure may be the initial presentation of ALL in an adolescent.
\end{abstract}

\section{Introduction}

Acute lymphoblastic leukemia (ALL) is the most common hematologic malignancy in the pediatric population, at least in the USA (1). The typical presentation includes signs and symptoms associated with bone marrow failure, including fever, infection, fatigue and excessive bruising. Liver involvement in ALL is a frequent phenomenon, but hyperbilirubinemia is not a frequently presenting symptom in ALL. Abnormal laboratory findings and their degree reflect the leukemic cell burden. However, there are few reports in the literature describing cases of ALL presenting with acute hepatic failure in a child or young adult (2-5). Acute hepatic failure is a relatively rare disease as an initial presentation of ALL in an otherwise healthy adolescent. This case report describes a case of an adolescent with ALL presenting with acute hepatic failure.

Correspondence to: Dr Jing Yuan, Department of Hematology, The Third Hospital of Hebei Medical University, 139 Ziqiang Road, Shijiazhuang, Hebei 050051, P.R. China

E-mail: yuanjing8886@163.com

Key words: acute lymphoblastic leukemia, liver failure, aminotransferase, malignancy

\section{Case report}

Six days prior to presentation, a previously healthy 15 -year-old male student began experiencing tiredness, inappetence, nausea, vomiting, and jaundice (yellow sclera and dark urine). Vomitus was nonbilious without hematemesis. There was no associated fever, cough, expectoration, abdominal pain, diarrhea, or other symptoms. The patient received a 3-day period of intravenous therapy at a Dingzhou People's Hospital (Dingzhou, China) between 6th of February and the 9th of February 2018, and he became increasingly jaundiced. He was then admitted to a primary care center for evaluation of his hyperbilirubinemia. The initial laboratory findings showed an alanine aminotransferase (ALT) of 492 IU/1, aspartate aminotransferase (AST) of $477 \mathrm{IU} / 1$, total bilirubin (TBIL) in serum of $11.7 \mathrm{mg} / \mathrm{dl}$, direct bilirubin (DBIL) of $7.4 \mathrm{mg} / \mathrm{dl}$, and lactic dehydrogenase ( $\mathrm{LDH})$ of $1,255 \mathrm{U} / 1$. The prothrombin time (PT) was $30.2 \mathrm{sec}$ and the prothrombin activity (PTA) was $13.4 \%$. The blood ammonia was $38.8 \mu \mathrm{mol} / 1$. The routine blood test showed that the patient's white blood cell count (WBC) was $2.41 \times 10^{9} / 1$ and the platelet (PLT) count was $49 \times 10^{9} / 1$. The patient's serology was negative for viral hepatitis. The patient underwent a 2-day transfusion treatment to protect the liver and lower transaminase levels. However, his physical condition progressively deteriorated and he was immediately transferred to the Third Hospital of Hebei Medical University (Shijiazhuang, China) on the 9th February 2018.

The patient was a student with sports talent. Further history of illness revealed that he had received a transfusion treatment 1 month previously due to a common cold. There was no history of hepatotoxic drugs or any other toxic agents. He had been healthy with no history of medical disorders or surgical procedures, and no history of taking medications. His family history was unremarkable for metabolic diseases or any other hereditary conditions. On physical examination, he had lymphadenectasis and splenectasis, and his vital signs were normal. No spider angioma or palmar erythema of chronic liver disease were present.

The laboratory data revealed a white blood cell count of $3.18 \times 10^{9} / 1$ (33.6\% neutrophils, $43.1 \%$ lymphocytes, $23.0 \%$ monocytes, and $0.3 \%$ eosinophils), hemoglobin level of $14.1 \mathrm{~g} / \mathrm{dl}$, hematocrit of $41.9 \%$, and PLT count of $52 \times 10^{9} / 1$. The metabolic panel revealed an ALT of 3,261 IU/1, AST of 2,110 IU/1, TBIL of $15.0 \mathrm{mg} / \mathrm{dl}$, DBIL of $11.1 \mathrm{mg} / \mathrm{dl}, \mathrm{LDH}$ of $1,767 \mathrm{U} / 1$, and alkaline phosphatase (ALP) of $175 \mathrm{IU} / \mathrm{l}$. The 
international normalized ratio was 2.66, PTA was $24.3 \%$, fibrinogen (FIB) was $0.86 \mathrm{~g} / \mathrm{l}$, PT was $31.2 \mathrm{sec}$, and activated partial thromboplastin time (APTT) was $40.1 \mathrm{sec}$. Urinalysis revealed urine was bilirubin-positive. A routine stool test and toxicological analysis were negative. A viral assessment for hepatitis A virus, hepatitis B virus (HBV), hepatitis C virus, hepatitis E virus, human immunodeficiency virus, Epstein-Barr virus, cytomegalovirus, Treponemapallidum and Salmonella typhi returned normal. An abdominal ultrasound with Doppler revealed splenomegaly, and the chest computed tomography scan was normal. Liver biopsy was not performed due to increased bleeding risk. Due to the evidence of decreased WBC and PLT count, lymphadenectasis and splenomegaly, a peripheral smear was obtained immediately. Atypical cells were observed by a hematologic pathologist as being consistent with hematologic malignancy. A bone marrow biopsy was then performed. The bone marrow cell morphology indicated hypercellularity, and the usual hematopoietic cells had been almost completely replaced by amonotonous lymphoid population composed mainly of lymphoblasts (Fig. 1A). The bone marrow core biopsy revealed large aggregates of blasts (Fig. 1B and C). Immunohistochemistry revealed the following:terminal deoxynucleotidyl transferase (TdT) (+), paired box 5 (+), CD34 (-), CD20 (-), CD3 (-), CD117 (-), myeloperoxidase (MPO) (-), and lysozyme (-). The cells did not show myeloperoxidase activity on cytochemical analysis. Bone marrow was submitted for flow cytometry, which revealed a predominant B-cell population (68.15\%) expressing CD19, cCD79a, CD33, CD38, CD123, CD9 and CD38, partially expressing CD10 and TdT, and showing weak expression of CD22, with no expression of CD117, CD34, CD7, CD15, CD64, CD11b, CD20, MPO, cCD3, mCD3, CD56, kappa or lambda. The diagnosis of early precursor B-cell (pro-B) ALL was established. In retrospect, the atypical cells of the initial peripheral blood smear were actually lymphoblasts, although the blood smear was not available for re-review following the diagnosis of ALL.

The patient underwent transfusion treatment at the Third Hospital of Hebei Medical University to protect the liver, including reduced glutathione injection (1.8 g per day for a total of 14 days), ornithine aspartate injection (2.0 g per day for a total of 14 days), compound diisopropylamine dichloroacetate injection (120 mg per day for a total of 14 days), and ademetionine 1,4-butanedisulfonate injection (1.0 g per day for a total of 14 days), and low-dose dexamethasone (10 mg per day for a total of 5 days). The levels of ALT, AST, LDH and TBIL continued to decrease, and the PTA and fibrinogen recovered (Table I). The patient presented with improvement of symptoms. Admission laboratory data revealed a WBC of $2.82 \times 10^{9} / 1$ (55\% neutrophils, $33 \%$ lymphocytes, $11 \%$ monocytes, $0.4 \%$ eosinophils and $0.6 \%$ basophils), hemoglobin of $91.3 \mathrm{~g} / 1$, TBIL of $6.9 \mathrm{mg} / \mathrm{dl}$, ALT of $95 \mathrm{IU} / 1$, AST of $46 \mathrm{IU} / 1$, and LDH of $124 \mathrm{U} / 1$. Due to persistent improvement in the patient's liver function tests, a liver biopsy was not necessary. Bone marrow cell morphology, flow cytometry and biopsy revealed young blasts with markers consistent with B-cell ALL. Cytogenetic examination indicated no abnormal chromosomes $(46, \mathrm{XY})$ or fusion genes, including breakpoint cluster region/ABL proto-oncogene 1 , non-receptor tyrosine kinase andmixed lineage leukemia. The adolescent was transferred to the Department of Hematology, where he was started on induction chemotherapy with pediatric-based protocols.

\section{Discussion}

ALL is the most common hematological neoplasm in the pediatric population, with a frequency peak for children between 2 and 5 years of age. The overall survival rate of ALL in childhood is approaching $90 \%$. By contrast, ALL is less common in adolescents and adults, with estimated survival rates between 20 and $40 \%(1,2)$. The common presenting signs and symptoms of ALL, although non-specific, include fever and infection caused by neutropenia, fatigue and pallor due to anemia, and bruising and hemorrhage secondary to thrombocytopenia. Other manifestations involve lymphadenectasis, hepatomegaly, splenomegaly, sternal tenderness, and joint pain. Hepatomegaly is a common feature of leukemia, however, acute hepatic failure as the initial presentation is uncommon. There are limited case reports in the literature describing ALL presenting as acute hepatic failure in an adolescent (3-5). Although rare, it is important to consider ALL in the differential diagnosis of multi-organ dysfunction, including acute liver failure, particularly in previously otherwise healthy individuals.

Several mechanisms have been proposed to explain the etiopathogenesis of acute hepatic failure in leukemia, including comorbidity with viral infections, particularly HBV, sepsis, autoimmune hepatitis, or hypoxia and ischemia caused by leukemic cell infiltration that results in the obstruction of hepatic blood flow. In the case described herein, on awaiting the results of bone marrow biopsy, hepatoprotective treatment was administered and the ALT, AST and TBIL levels slowly decreased. The biopsy revealed large aggregates of blasts, following which low-dose dexamethasone was initiated and serum levels of ALT, AST and TBIL showed accelerated decrease with marked improvement in the patient's physical condition. A limitation was that histological examination was not performed on the patient's liver. Therefore, it was not possible to demonstrate whether the elevated liver enzymes, hyperbilirubinemia, and hepatic failure were secondary to hepatic necrosis produced by leukemic infiltration. However, the early application of glucocorticoids was more effective than the hepatic protectant, which suggested that hepatic injury was likely caused by the infiltration of leukemic cells.

The majority of previous reports describing liver disease in patients with ALL are in the pediatric population. In one retrospective pediatric study which examined hepatitis at the time of ALL diagnosis, approximately one third of patients had increased aminotransferase levels, with normal bilirubin and ALP and no evidence of viral hepatitis. ALL-induced hepatitis was found to be more common in patients with T-cell ALL and in patients with higher WBC, uric acid, and LDH levels, suggesting leukemic cell infiltration as the underlying etiology (6). The mechanism of hepatic injury remains to be fully elucidated, but is likely to be either a paraneoplastic syndrome or due to lymphocyte infiltration of the liver. The patient's abnormal liver function tests in the case study reported normalized following the early application of dexamethasone. This indicated that lymphoblast infiltration 
Table I. Liver function and blood coagulation analyses.

\begin{tabular}{|c|c|c|c|c|c|c|c|}
\hline $\begin{array}{l}\text { Laboratory } \\
\text { parameter }\end{array}$ & $\begin{array}{c}\text { Normal } \\
\text { level }\end{array}$ & $\begin{array}{l}\text { Admission } \\
\text { (Day 1) }\end{array}$ & Day 3 & Day 5 & Day 10 & Day 12 & Day 18 \\
\hline ALT & $9-50 \mathrm{U} / 1$ & $3,261 \mathrm{U} / 1$ & $1,506 \mathrm{U} / 1$ & $694 \mathrm{U} / 1$ & $171 \mathrm{U} / 1$ & $120 \mathrm{U} / 1$ & $95 \mathrm{U} / 1$ \\
\hline AST & $15-40 \mathrm{U} / 1$ & $2,110 \mathrm{U} / 1$ & $880 \mathrm{U} / 1$ & $310 \mathrm{U} / 1$ & $35 \mathrm{U} / 1$ & $31 \mathrm{U} / 1$ & $46 \mathrm{U} / 1$ \\
\hline LDH & $120-250 \mathrm{U} / 1$ & $1,767 \mathrm{U} / 1$ & 794 U/1 & $606 \mathrm{U} / 1$ & $157 \mathrm{U} / 1$ & $123 \mathrm{U} / 1$ & $124 \mathrm{U} / 1$ \\
\hline TBIL & $3-20 \mu \mathrm{mol} / 1$ & $256 \mu \mathrm{mol} / 1$ & $274 \mu \mathrm{mol} / 1$ & $312 \mu \mathrm{mol} / 1$ & $196 \mu \mathrm{mol} / \mathrm{l}$ & $198 \mu \mathrm{mol} / 1$ & $118 \mu \mathrm{mol} / 1$ \\
\hline DBIL & $2-6 \mu \mathrm{mol} / 1$ & - & $205 \mu \mathrm{mol} / 1$ & $246 \mu \mathrm{mol} / 1$ & $164 \mu \mathrm{mol} / \mathrm{l}$ & $169 \mu \mathrm{mol} / 1$ & $101 \mu \mathrm{mol} / 1$ \\
\hline PT & $10-14 \mathrm{sec}$ & $31.2 \mathrm{sec}$ & $33.8 \mathrm{sec}$ & $24.5 \mathrm{sec}$ & $15.4 \mathrm{sec}$ & $11.8 \mathrm{sec}$ & $11.1 \mathrm{sec}$ \\
\hline PTA & $80-150 \%$ & $24.3 \%$ & $22.1 \%$ & $32.0 \%$ & $61.0 \%$ & $106 \%$ & $100 \%$ \\
\hline FIB & $2-4 \mathrm{~g} / 1$ & $0.86 \mathrm{~g} / 1$ & $0.61 \mathrm{~g} / 1$ & $0.83 \mathrm{~g} / 1$ & $0.60 \mathrm{~g} / 1$ & $1.29 \mathrm{~g} / 1$ & $3.47 \mathrm{~g} / 1$ \\
\hline
\end{tabular}

ALT, alanine aminotransferase; AST, aspartate aminotransferase; LDH, lactate dehydrogenase; TBIL, total bilirubin; DBIL direct bilirubin; PT, prothrombin time; PTA, prothrombin activity; FIB, fibrinogen.
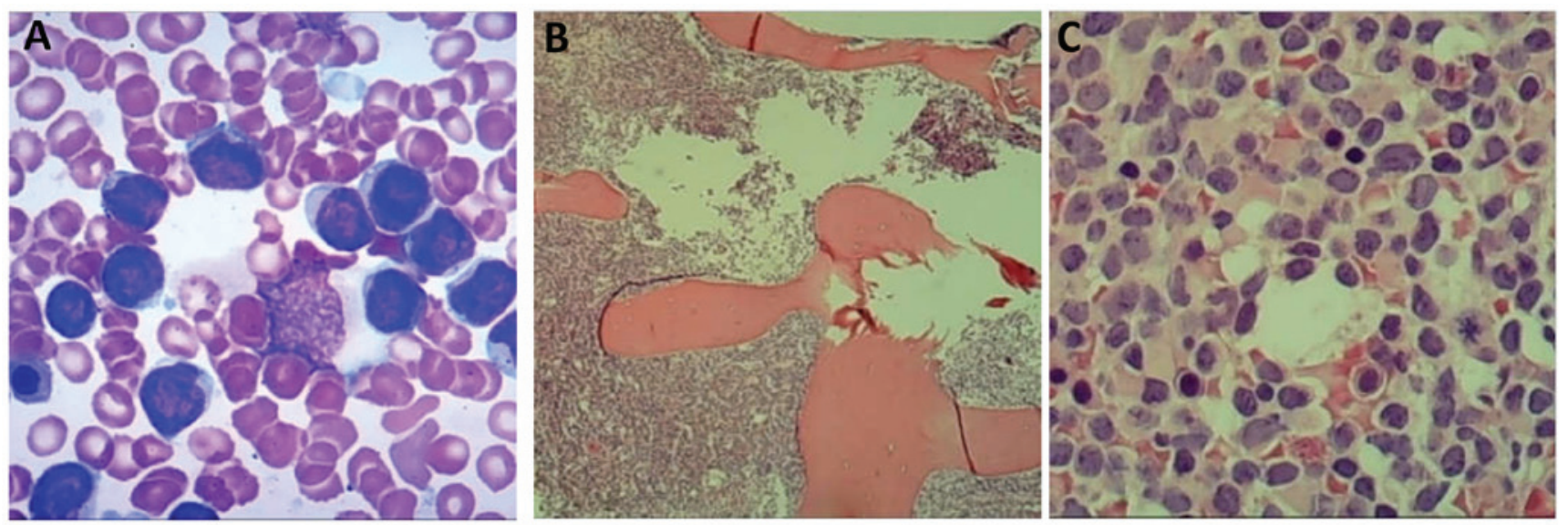

Figure 1. Bone marrow biopsy. (A) Bone-marrow smear demonstrating a predominance of intermediate-sized irregular cells with high nuclear to cytoplasmic ratios, fine nuclear chromatin, and variable prominent nucleoli, morphologically consistent with lymphoblasts (magnification, x1,000). (B) When visualized at a low magnification, the bone marrow core biopsy revealed large aggregates of blasts and reticular fiber staining was negative (magnification, x40). (C) Bone marrow core biopsy revealing large aggregates of blasts with reduced numbers of red and white blood cell precursors, consistent with a decrease in erythropoiesis and myelopoiesis (magnification, $\mathrm{x} 400$ ). Megakaryocytes were present in decreased numbers.

had led to the hepatic failure. The patient received induction chemotherapy according to pediatric-based protocols and without any dose adjustment due to liver dysfunction. This most likely resulted in an improvement in long-term survival rate (7). Taken together, it is important to consider ALL in the initial presentation of acute hepatic failure. A peripheral blood smear is useful for the differential diagnosis of hematological neoplasms, and bone marrow or liver biopsy is required as soon as possible for early diagnosis.

\section{Acknowledgements}

The authors would like to thank Dr Fei-yan Ma (Department of Ophthalmology, The Second Hospital of Hebei Medical University, Shijiazhuang, China) for her assistance in language editing.

\section{Funding}

No funding was received.

\section{Availability of data and materials}

Not applicable.

\section{Authors' contributions}

JY conceived and designed the study and collected clinical data. RLG, MX and JS collected clinical data and edited the figure. JY and RLG wrote the manuscript. All authors read and approved the final manuscript.

\section{Ethics approval and consent to participate}

The case report was approved by Institutional Review Board of the Third Affiliated Hospital of Hebei Medical University for chart review and data analysis.

\section{Patient consent for publication}

Patient consent was obtained for publication. 


\section{Competing interests}

The authors declare that they have no competing interests.

\section{References}

1. Inaba $\mathrm{H}$, Greaves $\mathrm{M}$ and Mullighan CG: Acute lymphoblastic leukaemia. Lancet 381: 1943-1955, 2013.

2. Vallacha A, Haider G, Raja W and Kumar D: Remission rate of acute lymphoblastic leukemia (ALL) in adolescents and young adults (AYA). J Coll Physicians Surg Pak 28: 118-121, 2018.

3. Litten JB, Rodríguez MM and Maniaci V: Acute lymphoblastic leukemia presenting in fulminant hepatic failure. Pediatr Blood Cancer 47: 842-845, 2006.
4. Kader A, Vara R, Egberongbe Y, Height S and Dhawan A: Leukaemia presenting with fulminant hepatic failure in a child Eur J Pediatr 163: 628-629, 2004.

5. Heincelman M, Karakala N and Rockey DC: Acute lymphoblastic leukemia in a young adult presenting as hepatitis and acute kidney injury. J Investig Med High Impact Case Rep: Sep 22, 2016 (Epub ahead of print). doi: 10.1177/2324709616665866.

6. Segal I, Rassekh SR, Bond MC, Senger C and Schreiber RA: Abnormal liver transaminases and conjugated hyperbilirubinemia at presentation of acute lymphoblastic leukemia. Pediatr Blood Cancer : 434-439, 2010.

7. Ribera JM, Ribera J and Genescà E: Treatment of adolescent and young adults with acute lymphoblastic leukemia. Mediterr J Hematol Infect Dis 6: e2014052, 2014. 